\title{
Dense Active Appearance Models Using a Bounded Diameter Minimum Spanning Tree
}

\author{
Robert Anderson ${ }^{1}$ \\ ra312@cam.ac.uk \\ Bjorn Stenger ${ }^{2}$ \\ bjorn.stenger@crl.toshiba.co.uk \\ Roberto Cipolla ${ }^{1}$ \\ cipolla@eng.cam.ac.uk
}

\author{
${ }^{1}$ Department of Engineering \\ Cambridge University \\ Cambridge, UK \\ ${ }^{2}$ Cambridge Research Laboratory \\ Toshiba Research Europe \\ Cambridge, UK
}

\begin{abstract}
We present a method for producing dense Active Appearance Models (AAMs), suitable for video-realistic synthesis. To this end we estimate a joint alignment of all training images using a set of pairwise registrations and ensure that these pairwise registrations are only calculated between similar images. This is achieved by defining a graph on the image set whose edge weights correspond to registration errors and computing a bounded diameter minimum spanning tree (BDMST). Dense optical flow is used to compute pairwise registration and we introduce a flow refinement method to align small scale texture. Once registration between training images has been established we propose a method to add vertices to the AAM in a way that minimises error between the observed flow fields and a flow field interpolated between the AAM mesh points. We demonstrate a significant improvement in model compactness using the proposed method and show it dealing with cases that are problematic for current state-of-the-art approaches.
\end{abstract}

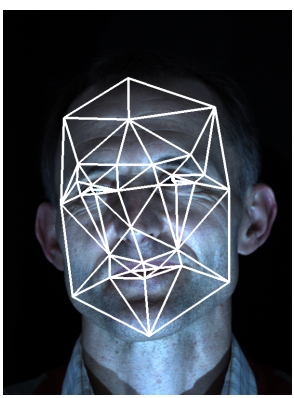

(a)

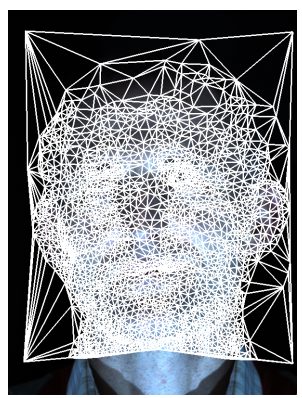

(b)

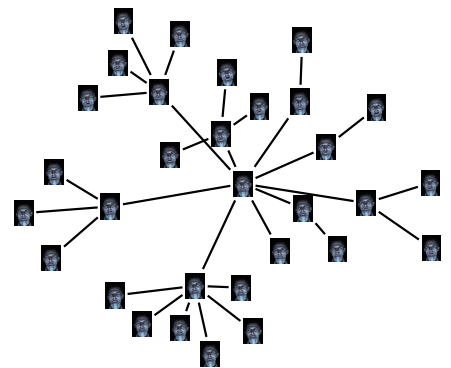

(c)

Figure 1: Overview: From a sparse, 37 point AAM (a) the proposed method creates a dense, 1000 point AAM (b) suitable for image synthesis tasks. To achieve the dense registration required to construct the AAM we find a bounded diameter minimum spanning tree on a graph defined on the training images (c). On the digital version it is possible to zoom in to see the individual training images. 


\section{Introduction}

Active Appearance Models (AAMs) are statistical models of both shape and appearance. Since their introduction more than ten years ago [日], they have been used for tracking as they allow robust and efficient registration [四]. More recently, AAMs have been growing in popularity for synthesis, for example in emotion synthesis [], expression transfer [ $\square]$ ] and visual text-to-speech applications [ $\square]$. In order to train an AAM, a set of points must be consistently labelled in a collection of training images. Since this is usually carried out by hand the number of points is small $(<100)$, leading to models such as the one in figure 1(a). While automatic model building methods have been proposed previously $[\mathbf{Q}, \mathbf{U}]$ ] these do not produce results of sufficient accuracy for the synthesis of high-resolution images.

The task addressed in this paper is building dense AAMs, such as the example shown in figure 1(b), in order to generate new video-realistic synthetic sequences. The underlying problem that needs to be solved in order to build such models is one of joint non-rigid image alignment. There exists a large body of work on this problem $[\square, \boldsymbol{\square}, \mathbb{\square}, \mathbb{Q}$, , the majority of which registers each image to an iteratively updated model. In this paper we propose a method that instead of registering all images to a base model registers images in a pairwise fashion. We find a bounded diameter minimum spanning tree (BDMST) on a graph of all the images, where each image is a node in a graph and each edge represents a warp calculated between the two images it connects (see figure 1(c)). The motivation for this approach is the fact that with current pairwise registration methods a low alignment error can only be achieved between similar images. Given the spanning tree all images can be registered to a common reference frame, solving the joint alignment problem.

In this paper we use a dense optical flow algorithm to align two images. Current state-ofthe-art methods use a coarse-to-fine approach, which often fails to register small scale texture. We therefore introduce an optical flow refinement technique that is particularly suited to registering regions containing only fine texture. Once the joint alignment is computed we present a method for densifying AAMs that is based on established ideas from digital terrain modelling. To summarise, the contributions of this paper are:

1. an application of bounded diameter minimum spanning trees to the joint alignment problem,

2. a method of optical flow refinement suited to regions which contain fine texture, and

3. a method for densifying an AAM given dense correspondences between training images.

The paper is organised as follows: $\$ 1.1$ reviews prior work and $\$ 2$ gives the motivation for our approach. The use of the BDMST is described in $\S 3$, with the details of the optical flow algorithm presented in $\S 4$ and details of how the AAM is densified given the joint alignment in $§ 5$. $\S 6$ shows the effect of different parameter settings, compares the proposed technique with competing methods and demonstrates the effectiveness of our approach for the AAM synthesis task.

\subsection{Related Work}

There exists a significant body of work on automating the process of registering images for model building. The two main approaches are (1) to iteratively refine the model itself [ $[$, 


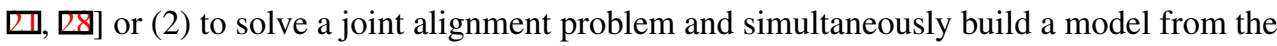
registered images $[\square, \nabla]$. We briefly review each of these.

Model-based approaches The work of Vetter et al. [R] builds linear models automatically by using optical flow to register each new training image to the closest image that can be generated by the current linear model. The same technique has been applied to automatically building morphable models [ [ $]$ ].

Automatic AAM construction was formulated as an image coding problem by Baker et al. [ []]. However, it was only demonstrated on a dataset with little nonrigid deformation. Ramnath et al. [ $[\mathbb{U}]$ incrementally densify an AAM by using standard AAM fitting techniques and allowing for shape modes spanning the whole space of possible motions. This technique produces good results but is dependent on the initial input mesh as points are penalised for moving from their hand labelled positions.

While most techniques work on images given in an arbitrary order, some are designed specifically for ordered sequences where temporal constraints can be used [ $\mathbb{Z}, \mathbf{Q}]$. The problem can then be treated as tracking with an adaptive template. These approaches have the advantage of placing additional constraints on the registration problem, but they are not applicable to all scenarios.

Joint alignment approaches There are a large number of methods for pairwise image registration, for a survey see [B]]. It has been shown, however, that there is often an advantage in registering sets of images jointly instead of in a pairwise manner [ब], taking advantage of all the information present in the image data. Current state-of-the-art results for automatic model building are achieved by performing joint image alignment and warping points from a reference frame onto all images [ $\square$, Q $]$. Cootes et al. [ $[$ ] formulate joint image alignment as a Minimum Description Length encoding problem in which an efficient encoding of the image set represents a good registration. This approach produces very good results, however if features only appear in a small subset of the images then problems arise since all images are registered to a mean image which may not contain these features. Marsland et al. [ㅁ] ] demonstrate an iterative joint alignment process in which all training images are compared to one of the training images instead of their mean. The choice of this reference image may change during the joint alignment process. However, even the optimal choice of reference image may be unsuitable for data sets exhibiting significant variation. Sidirov et al. []] have demonstrated good results by posing joint image alignment as a large-scale optimisation problem, minimising pixel discrepancy between all registered images, which is solved using stochastic techniques. More recently the method was extended to registering texture mapped surfaces [Q⿴囗⿰丿㇄心].

Some types of tree have already been used in the joint alignment problem, for example Cristinacce and Cootes [ $[$ ] use a shortest path tree over clusters of images. They use the tree to determine what order images are added to a joint alignment rather than concatenating deformations as we do. The most similar approaches to the one proposed are the geodesic methods from the medical literature. For example Hamm et al. [ $\square$ ] use a k-nearest neighbour graph to approximate a manifold upon which valid images lie. Images are then registered by concatenating diffeomorphisms along the shortest path in the tree as an approximation to following the shortest geodesic distance over this manifold. Minimum spanning trees have also been used for registration in the medical literature as a method of estimating Rényi entropy [ㅁ]]. 


\section{Approach Overview}

We densify AAMs using a two-stage process; first a joint registration is found between all of the training images $\left\{I_{i}\right\}_{i=1}^{N}$, then additional mesh vertices are added automatically to one training image and propagated to all others using the joint registration. In order to find the joint registration between all training images we calculate pairwise registrations between selected training images in the form of flow fields. All images are registered to a common frame by concatenating these flow fields.

The advantage of using pairwise registration is that when the correct image pairs are chosen each image is only registered with images that are similar in appearance. This is particularly important when there are features that only appear in a few images as it ensures that these features are well registered between the images in which they appear, whereas if these images were registered to a mean image lacking these features then registration is likely to fail in these regions.

\section{Choice of Registrations to Compute}

Joint registration through concatenating pairwise flows can be viewed in a graphical form. Each node in the graph represents an image and each edge represents a dense mapping computed between two images. To be able to jointly align all of the images a spanning tree must be found so that all of the images are part of the same connected graph.

In order to minimise errors in registration we wish to register between images that are as similar as possible. We assign each edge a cost $C_{i j}$ for registering images $I_{i}$ and $I_{j}$ where $C_{i j}$ is a measure of error between the two images after alignment. In order to register all images whilst minimising the sum of the pairwise registration errors we can now find a minimum spanning tree of the graph.

The problem with using a standard minimum spanning tree is that to register all images to a common image it may be necessary to concatenate a large number of flow fields along a path. This leads to error accumulation and results in a poor joint alignment. In order to limit the number of flow fields that need to be concatenated to reach the reference image from any other image we place a bound on the diameter of our minimum spanning tree. The diameter $D$ is defined as the largest number of edges in any path between any two nodes in the spanning tree, for example the minimum spanning tree in figure 2(a) has a diameter of 10 edges. The tree in figure 1(c) on the other hand has a diameter of 4 , leading to lower accumulation of error when all of the images are registered to a single frame. We register all images to the image at the root of the tree, requiring concatenation of at most $\frac{D}{2}$ flow fields to register to this image.

Choosing which pairwise registrations to compute now becomes a case of choosing a diameter $D$ and finding a corresponding BDMST.

\subsection{Finding a Bounded Diameter Minimum Spanning Tree}

The theory of BDMSTs has been well studied in the graph literature. For trees containing $N$ nodes and with a diameter of $D$, where $4 \leq D<N-1$, finding an optimal tree is NP-hard [ $\square]$ and hence a number of heuristic methods have been proposed $[\square, \mathbb{Q} \theta]$. For trees with a small diameter it has been shown that randomised approaches give better results than purely greedy ones $[\square]$. This motivates our use of the randomised centre-based tree reconstruction 


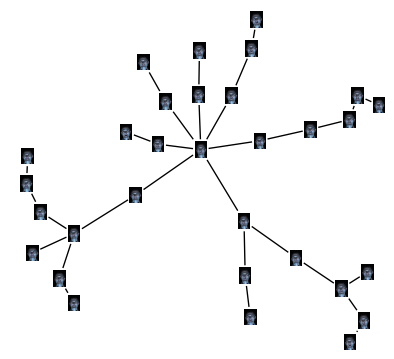

(a)

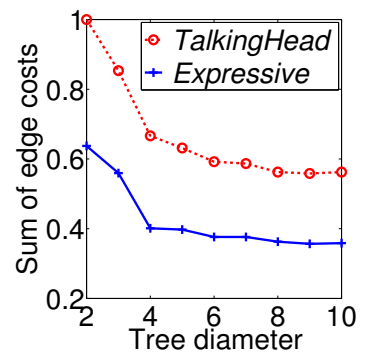

(b)

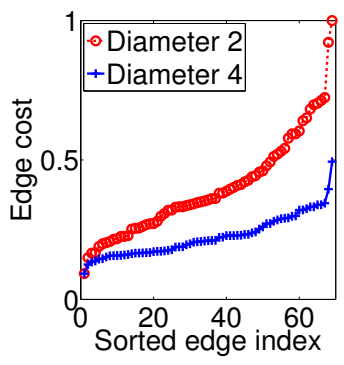

(c)

Figure 2: Effects of changing the tree diameter. (a) A minimum spanning tree with diameter $D=10$ of the Expressive dataset. (b) Sum of edge costs for a range of minimum spanning tree diameters. (c) Individual edge costs in a minimum spanning tree of diameter 2 (red) and diameter 4 (blue) arranged in ascending order.

algorithm proposed by Julstrom [ㅁ] . This method repeatedly constructs BDMSTs in a semirandomised way and retains the tree with the lowest sum of edge weights.

To allow a tree to be built we need a measure of how well two images can be registered. To approximate this cost we register the two images in question to the same frame using optical flow and then take the $l^{2}$-norm of the image difference. In order to make the cost symmetrical we sum the scores achieved by warping to both image $I_{i}$ and image $I_{j}$ :

$$
C_{i j}=\left|I_{i}-W_{i j}\left(I_{j}\right)\right|^{2}+\left|I_{j}-W_{j i}\left(I_{i}\right)\right|^{2}
$$

where $W_{i j}$ represents the warp from image $I_{j}$ to $I_{i}$ computed by optical flow.

\subsection{Choice of Tree Diameter}

Figure 2(b) shows the total weight of all the edges in a spanning tree calculated for different diameters using the method above. The input comes from 2 different datasets, TalkingHead and Expressive, details of which are given in the results section. It can be seen that the decrease in the sum of the edge costs and hence the gain made through registering similar images starts to drop off rapidly after $D=4$. This suggests limiting the tree diameter to 4 and comparisons in the results section for models built using trees of different diameter also support this choice.

Figure 2(c) shows the edge weights for one of the datasets for a graph with a diameter of $D=2$ (which corresponds to registering all images to one base image) and $D=4$. It can be seen that the average edge cost is lower for the case where $D=4$, indicating that each individual registration problem has a significantly lower error in this case.

\section{Optical Flow Refinement}

To register images we choose dense flow fields as a transformation model. While significant advances have been made in the development of robust optical flow algorithms, one of their drawbacks is that the commonly employed coarse-to-fine approach does not allow for the registration of structures which are displaced by a distance greater than their own size, due to the structure being completely blurred away at the pyramid level that would allow its matching [Q]. To build an accurate, dense AAM we need to register structures that are only a few pixels in size but which may be displaced by several pixels within the training images. 
To overcome this limitation we therefore propose a two-stage approach. An initial estimate of the flow is computed using a modern optical flow algorithm and is used to approximately align two input images; we use the implementation of Liu []]. A refinement step is then carried out calculating flow between the two partially aligned images over a small range of $\pm r$ pixels, where we have empirically set $r=15$.

We formulate the flow refinement problem as a Markov Random Field (MRF) optimisation. Since we allow for displacements of $\pm r$ pixels in both the horizontal and vertical directions this results in $(2 r+1)^{2}$ possible integer displacements. Optimising simultaneously over all displacements would result in a number of labels that is too large for current MRF optimisers. To make the problem computationally tractable, we estimate the horizontal and vertical components of the flow field independently. In the following we outline the method for solving for horizontal flow $\mathbf{u}$, the method for calculating vertical flow being analogous. We define an MRF with one node per pixel, connected in a 4-neighbourhood with the following energy terms. The pairwise term $P_{i j}$ between two connected nodes, $i$ and $j$, takes the form of a truncated quadratic,

$$
P_{i j}=\alpha \min \left(\left|u_{i}-u_{j}\right|^{2}, P_{\max }\right),
$$

where $P_{\text {max }}$ is the point at which the quadratic is truncated and $\alpha$ is a weighting term. This pairwise term encourages piecewise smoothness. The unary term, $U_{(a, b)}$, for a pixel in column $a$ and row $b$ is given by

$$
U_{(a, b)}=\min \left(\min _{-r \leq v \leq r}\left(\sum_{x=a-w}^{a+w} \sum_{y=b-w}^{b+w}\left|I_{1}(x, y)-I_{2}(x+u, y+v)\right|^{2}\right), U_{\max }\right) .
$$

This is the minimum SSD error, over a window of width $2 w+1$, that can be achieved for a horizontal displacement of $u$ for any vertical displacement $v$ within the allowed range of displacements $\pm r$. This metric is made robust by thresholding the maximum cost at $U_{\max }$. In order to efficiently compute the SSD scores for all images we take advantage of integral images. The constants were set empirically and were fixed at $\alpha=100, w=7, U_{\max }=2500$ and $P_{\max }=25$ for all experiments. The tree-reweighted message passing algorithm is used

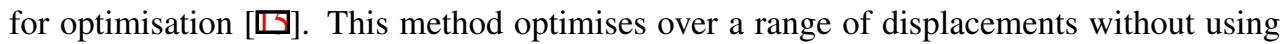
any smoothing allowing for the alignment of fine texture. In our experience, the global nature of the MRF avoids the local minima created by the separation of the horizontal and vertical flow components.

\section{Mesh Densification}

Once correspondence has been established between all training images, additional vertices are added to the model in order to increase its descriptive power. We aim to add points in a way that minimises the difference between the flow fields we have calculated and those which the mesh is able to model by using linear interpolation of flow between vertices. We aim to minimise at each pixel $p$ the error function

$$
E_{p}=\sum_{i=1}^{N} w_{p}\left|\mathbf{x}_{i}-\mathbf{y}_{i}\right|^{2}
$$

where $\mathbf{y}_{i}$ is the linear approximation to the flow field for training image $i$ provided by the mesh, $\mathbf{x}_{i}$ is the calculated flow field for image $i$ and $w_{p}$ is a per-pixel weight. The weight 


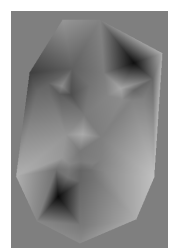

(a)

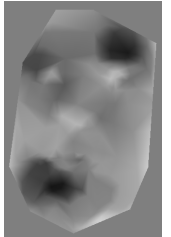

(b)

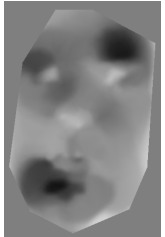

(c)

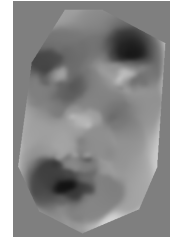

(d)

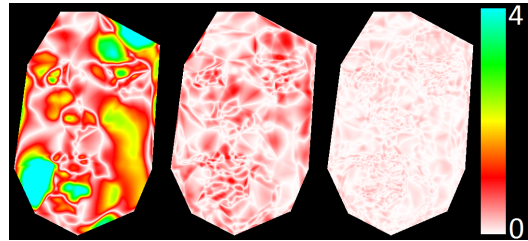

(e)

Figure 3: Flow field improvement by AAM densification. (a-c) Approximated horizontal flow fields for one image using meshes with 50, 250 and 1000 vertices respectively, (d) target horizontal flow field, (e) errors between the target flow field and approximations using 50, 250 and 1000 vertices.

$w_{p}$ is used to increase the mesh density in regions with a lot of variation in the images and decrease it in smooth regions, such as the background. It is given by the sum of the local variation of each training image warped into the base image's reference frame.

In order to solve this problem we draw on ideas from the construction of digital terrain models. Instead of aiming to approximate a scalar height field we aim to approximate a vector field formed by pixelwise concatenation of the $2 \mathrm{D}$ flow vectors of all training images.

We use the established, greedy method of DeFlorian [ $[$ ] to solve this problem: The original vertices are used as an initial mesh and an approximation to the flow fields is constructed using linear interpolation between these points. Vertices are added iteratively at the point with the greatest error $E_{p}$ and the mesh approximation to the flow fields is recalculated. Since this involves only triangles containing the inserted point this is an efficient local operation.

Figure 3 shows an example of the evolution of one component of a single flow field as the number of vertices is increased. We continue the densification until a fixed number of points have been added.

\section{Experiments}

To evaluate our approach we use two datasets, both consisting of images of $800 \times 600$ resolution of a single subject:

1. TalkingHead - A dataset of 70 images. The AAMs built for this model are used to track an hour long sequence and train a 'talking head' model. The initial sparse AAM has 53 points.

2. Expressive - A sequence of 31 images demonstrating a much greater degree of expressiveness than the TalkingHead dataset. The initial sparse AAM has 37 points.

In order to compare the resulting models we use the model compactness measure used in [四]. This approximates the compactness of a Gaussian distributed model by the determinant of the model's covariance matrix, where a low value corresponds to a more compact model. Since the shape model component of the AAM is significantly more compact than the texture model we only report texture model compactness. In all cases the model compactness is scaled so that the least compact model has a compactness score of 1 .

Before any processing we first high pass filter all input images to reduce the effect of lighting variation. We subsequently warp all images into approximate correspondence using the initial hand-labelled sparse AAM points, making no further use of the hand labelling after this point. 


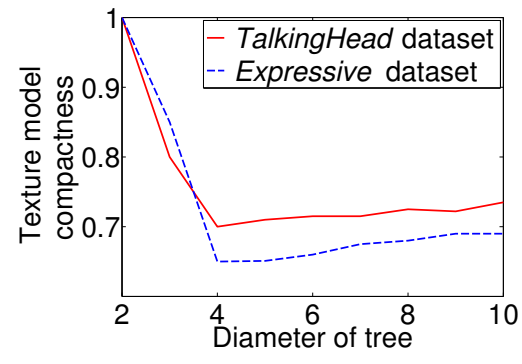

(a)

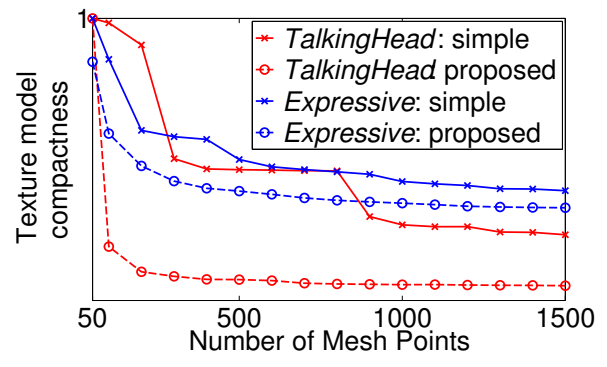

(b)

Figure 4: Model compactness for varying parameters. Graphs showing the effect of (a) varying tree diameter and (b) varying mesh density with simple densification (crosses) and the proposed densification (circles). Lower is better.

Effect of Tree Diameter Figure 4(a) shows the effect of tree diameter on model compactness. There is a sharp decrease between a diameter of $D=2$, corresponding to registering all images to the same base image, and $D=4$, demonstrating that the spanning tree allows for more accurate registration. Note that a diameter value of $D=4$ allows clusters of similar images to form (as seen in figure 1(c)). Beyond this diameter value there is a slow increase in error as the paths in the tree become longer, leading to accumulation of error when concatenating flows.

Effect of Densification Method In order to demonstrate the effectiveness of the proposed mesh densification method presented in section 5, we compare it to a simple densification approach where at each iteration a vertex is added at the centre of the largest triangle and then the mesh is re-triangulated. An example of a model densified using the proposed method can be seen in in figure1(b). Note the increased mesh density in areas which exhibit significant deformation, such as regions around the eyes, as opposed to more rigid regions like the nose. As can be seen from figure 4(b) for both datasets the proposed method results in a more compact model with fewer mesh points.

Effect of Optical Flow Refinement To demonstrate the value of the proposed optical flow refinement method we compare it to the flow method of [ $\square$ ], which we use for initialisation. Due to the challenge of obtaining ground truth data for this type of non-rigid flow problem we set up the following experiment. Each image in the Expressive dataset is warped by a flow field calculated between two other random images in the data set. This procedure yields a set of warped images with known flow fields. Gaussian noise is added to the images and we then apply the flow method of [ $\square$ ], with and without the refinement proposed in section 4, and measure the per-pixel error in flow fields for the face region. Figure 5(a) shows the resulting errors as a cumulative percentage of pixels with errors below a given threshold. It can be seen that flow refinement results in significant error reduction, e.g. the percentage of pixels with a $<1$ pixel error increased from $73 \%$ to $87 \%$. This improvement in flow also leads to more compact models as fine texture is better aligned. In a separate experiment we find that flow refinement reduces the model compactness score by $17 \%$ on the Expressive dataset and $26 \%$ on the TalkingHead dataset. 


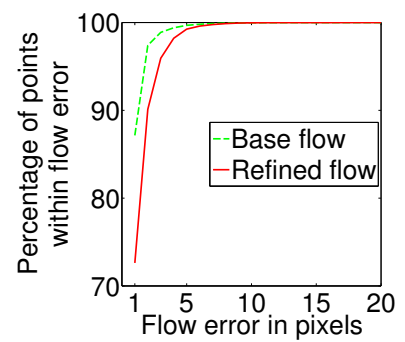

(a)

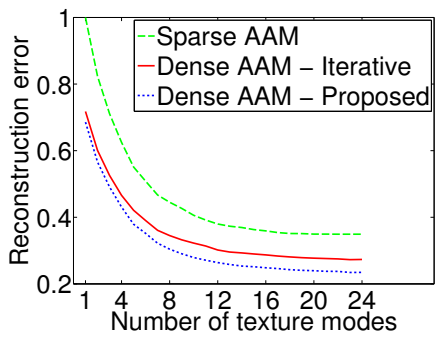

(b)

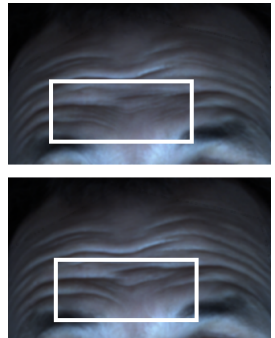

(c)

Figure 5: Effects of flow refinement and varying number of AAM modes. (a) Percentage of pixels for which flow was correctly calculated to within a given error, using the flow of [ $\square$ ] with (green) and without (red) our refinement. (b) Errors in reconstruction of a sequence using an AAM built using different approaches. (c) One frame of synthesis using an AAM built using iterative registration to a mean image (top) and the proposed method (bottom).

\subsection{Synthesis of an Expressive Sequence}

To demonstrate the advantage of using dense AAMs for synthesis we build an AAM of the Expressive dataset and use it to track a 450 frame long sequence and resynthesise it. We compare the original sparse model, a dense model built using the proposed method, and a dense model built using the popular method of jointly aligning all images to an iteratively updated mean image (similar to $[\boldsymbol{Q}]$ ). The dense models were constrained to have the same convex hull as the original model. Figure 5(b) shows the $L^{2}$-norm of the errors between the original image sequence that was tracked and the synthesised sequences. There is a large increase in accuracy when using a dense AAM instead of a sparse one. Figure 5(c) shows a close-up view of one the synthesised frames in which the model built by registering to a mean image (top) produces blending artefacts due to poor registration of the wrinkles that are only present in two of the training images. The model built using the proposed method shows no blending artefacts (bottom).

\subsection{Using a Dense AAM for a Talking Head}

One application of dense AAMs is in visual text-to-speech systems. To demonstrate the advantage of a dense AAM we train a 'talking head' model using a sparse AAM and a dense AAM built using the proposed method. As can be seen in figure 6 and the supplementary

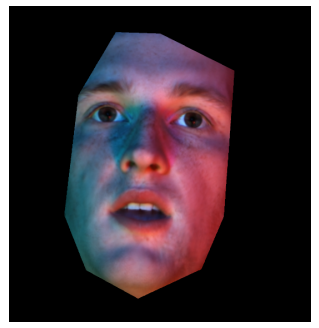

(a)

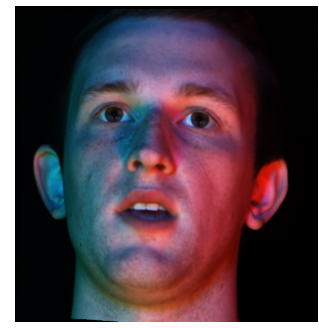

(b)

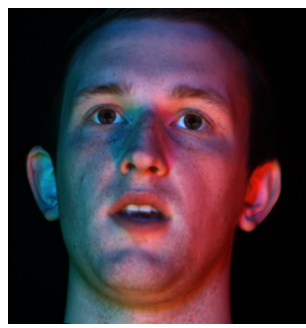

(c)

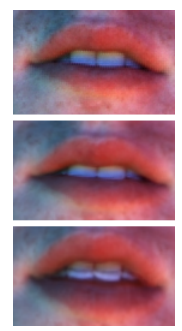

(d)

Figure 6: Synthesising novel speech with a virtual talking head. The same frame of synthesis using (a) the original sparse AAM, (b) a dense AAM built by densifying the sparse AAM and (c) a dense AAM built fully automatically. (d) top - the mouth region of a target image, middle - reconstruction using densified AAM, bottom - reconstruction using AAM built fully automatically. The model built fully automatically exhibits reconstruction artefacts. 
video the dense AAM is significantly sharper. We also built a dense AAM without providing an initial sparse mesh. This resulted in good results in most regions (figure 6(c)) but was not able to fully handle the complex occlusions and disocclusions around the mouth, resulting in artefacts during synthesis (figure 6(d)). Initial labelling in this region is currently still required in order to produce high-quality models.

\section{Conclusions}

We have shown that concatenation of pairwise registrations can give good results on the joint image alignment problem when the correct registrations are chosen. We have demonstrated the use of a BDMST as a method for choosing these registrations. An MRF-based optical flow refinement technique and a method for mesh densification were demonstrated to improve the results in terms of model compactness. As a target application we have shown synthesis results of person specific active appearance models.

\section{References}

[1] B. Abboud, F. Davoine, and M. Dang. Facial expression recognition and synthesis based on an appearance model. Signal Process.: Image Commun., 19(8):723-740, 2004.

[2] A. Abdalla, N. Deo, and P. Gupta. Random-tree diameter and the diameter constrained MST. Congressus Numerantium, pages 161-182, 2000.

[3] S. Baker, I. Matthews, and J. Schneider. Automatic construction of active appearance models as an image coding problem. PAMI, 26(10):1380-1384, 2004.

[4] V. Blanz and T. Vetter. A morphable model for the synthesis of 3D faces. In SIGGRAPH, pages 187-194, 1999.

[5] T. Brox and C. Bregler. Large displacement optical flow. CVPR, pages 41-48, 2009.

[6] T. Cootes, G. Edwards, and C. Taylor. Active appearance models. ECCV, 2:484-498, 1998.

[7] T. Cootes, C. Twining, V. Petrović, R. Schestowitz, and C. Taylor. Groupwise construction of appearance models using piece-wise affine deformations. BMVC, pages $879-$ $888,2005$.

[8] T. Cootes, C. Twining, V. Petrović, K. Babalola, and C. Taylor. Computing accurate correspondences across groups of images. PAMI, 32(11):1994-2005, 2010.

[9] D. Cristinacce and T. Cootes. Facial motion analysis using clustered shortest path tree registration. MLVMA, 2008.

[10] L. De Floriani. A pyramidal data structure for triangle-based surface description. IEEE Comput. Graph. Appl., 9(2):67-78, 1989.

[11] S. Deena, S. Hou, and A. Galata. Visual speech synthesis by modelling coarticulation dynamics using a non-parametric switching state-space model. ICMI-MLMI, pages $1-8,2010$. 
[12] M. Garey and D. Johnson. Computers and Intractability: A Guide to the Theory of NP-Completeness. Freeman, San Francisco, 1979.

[13] J. Hamm, D. Hye Ye, R. Verma, and C. Davatzikos. Gram: A framework for geodesic registration on anatomical manifolds. Medical Image Analysis, 14(5):633-642, 2010.

[14] B. Julstrom. Greedy heuristics for the bounded diameter minimum spanning tree problem. J. Exp. Algorithmics, 14:1:1.1-1:1.14, 2009.

[15] V. Kolmogorov. Convergent tree-reweighted message passing for energy minimization. PAMI, 28(10):1568-1583, 2006.

[16] E. Learned-Miller. Data driven image models through continuous joint alignment. PAMI, 28(2):236-250, 2006.

[17] C. Liu. Beyond pixels: Exploring new representations and applications for motion analysis. Doctoral Thesis, MIT, 2009.

[18] B. Ma, A. Hero, J. Gorman, and O. Michel. Image registration with minimum spanning tree algorithm. International Conference on Image Processing, 1:481 - 484, 2000.

[19] S. Marsland, C. Twining, and C. Taylor. Groupwise non-rigid registration using polyharmonic clamped-plate splines. MICCAI, 2879:771-779, 2003.

[20] I. Matthews and S. Baker. Active appearance models revisited. IJCV, 60(2):135-164, 2004.

[21] K. Ramnath, S. Baker, I. Matthews, and D. Ramanan. Increasing the density of active appearance models. CVPR, 2008.

[22] J. Saragih and R. Goecke. Learning active appearance models from image sequences. HCSNet workshop on Use of vision in human-computer interaction, pages 51-60, 2006.

[23] J. Saragih and R. Goecke. Monocular and stereo methods for aam learning from video. CVPR, 2007.

[24] K. Sidorov, S. Richmond, and D. Marshall. An efficient stochastic approach to groupwise non-rigid image registration. CVPR, pages $2208-2213,2009$.

[25] K. Sidorov, S. Richmond, and D. Marshall. Efficient groupwise non-rigid registration of textured surfaces. CVPR, pages 2401-2408, 2011.

[26] A. Singh and A. Gupta. Improved heuristics for the bounded-diameter minimum spanning tree problem. Soft Computing11, pages 911 - 921, 2007.

[27] B. Theobald, I. Matthews, J. Cohn, and S. Boker. Real-time expression cloning using appearance models. ICMI, pages 134-139, 2007.

[28] T. Vetter, M. Jones, and T. Poggio. A bootstrapping algorithm for learning linear models of object classes. CVPR, pages 40-46, 1997.

[29] K. Walker, T. Cootes, and C. Taylor. Automatically building appearance models from image sequences using salient features. BMVC, pages 463-562, 1999.

[30] B. Zitova and J. Flusser. Image registration methods: a survey. Image and Vision Computing, 21(11):977-1000, 2003. 